\title{
Copper-Promoted $N$-Arylations of Cyclic Imides within Six-Membered Rings: A Facile Route to Arylene-Based Organic Materials
}

\author{
Erin T. Chernick, Michael J. Ahrens, Karl A. Scheidt*, Michael R. Wasielewski* \\ Northwestern University, Department of Chemistry and Center for Nanofabrication and \\ Molecular Self-Assembly, 2145 Sheridan Road, Evanston, Illinois, 60208-3113
}

\section{Contents}

\section{Supporting Information}

General Experimental Details (S1-S2)

General Reaction Conditions (S2)

Experimental Details and full characterization for compounds 2d, 7, 10, and 11 (S2-S6)

${ }^{1} \mathrm{H}$ NMR spectra for compounds 2d, 7, 10, and 11 (S6-S9)

References (S10)

General: Reagents were purchased from commercial suppliers and used without further purification. Proton nuclear magnetic resonance spectra ( ${ }^{1} \mathrm{H}$ NMR) were taken on a 400 $\mathrm{MHz}$ or $500 \mathrm{MHz}$ spectrometer. TMS was used as an internal standard for all ${ }^{1} \mathrm{H}$ NMR. Laser desorption mass spectra were obtained with a time-of-flight MALDI mass spectrometer using dithranol as a matrix. High-resolution fast atom bombardment (HR FAB) measurements were made at the University Illinois at Urbana-Champaign. All column chromatography was performed on standard silica gel, $60 \AA$, 32-63 $\mu \mathrm{m}$. Methylene chloride was unstabilized and obtained from a column solvent system in which it is deoxygenated and passed through two alumina columns to remove any water. ${ }^{1}$

HPLC measurements were obtained using Phenomenex (4.6 x $250 \mathrm{~mm}$ x $5 \mu \mathrm{m})$ silica column. All samples were filtered with a $0.2 \mu \mathrm{m}$ syringe filter prior to injection into the HPLC. An eluent mixture of $80 \%$ hexanes to $20 \%$ ethyl acetate, both of which

1. Pangborn, A. B.; Giardello, M. A.; Grubbs, R. H.; Rosen, R. K.; Timmers, F. J. Organometal. 1996, 15, 1518-1520. 
were HPLC grade was used for the mobile phase. Compound $\mathbf{3}$ was used as an external standard from which reaction yields were obtained.

\section{General Reaction Conditions for Imide Formation}

A $20 \mathrm{~mL}$ reaction vial is flame dried and cooled under nitrogen to which $4 \AA$ molecular sieves, $2 \mathrm{~mL}$ of dry $\mathrm{CH}_{2} \mathrm{Cl}_{2}$, a $5 \mathrm{~mm}$ stir bar, $0.5 \mathrm{mmol}$ (2-3 equiv) of boronic ester, $0.17 \mathrm{mmol}$ ( 1 equiv) of imide, $0.5 \mathrm{mmol}$ ( 3 equiv) triethylamine, and 0.3-0.5 mmol of copper(II) acetate is added. The septum on the reaction vial is pierced with a needle connected to a balloon filled with oxygen. The atmosphere is flushed with oxygen and then the vial is sealed. The reaction is stirred for $19-20$ hours with gentle heating $\left(45^{\circ} \mathrm{C}\right)$. Upon consumption of the starting material imide, as judged by HPLC, the reaction mixture was purified directly via flash column chromatography on silica gel, usually using methylene chloride as the eluent.

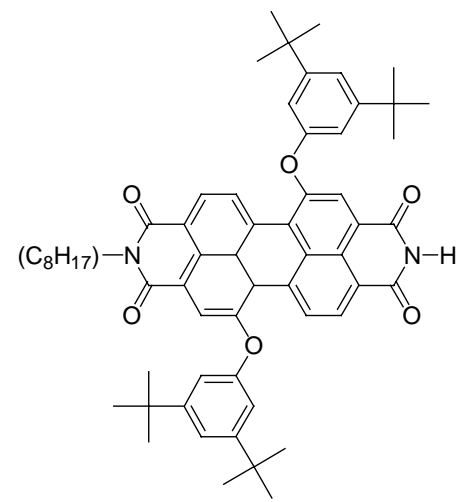

Compound 2d, 5,12-Bis-(3,5-di-tert-butyl-phenoxy)-2-octyl-5a,14c-dihydro-anthra[2,1,9def;6,5,10-d'e'f']diisoquinoline-1,3,8,10-tetraone.

In a flame dried $100 \mathrm{~mL}$ round bottom flask was added $N$-(octyl)-1,7-(3',5'-di-tbutylphenoxy)perylene-3,4-dicarboxyanhydride-9,10-dicarboximide $(0.11 \mathrm{~g}, 0.12 \mathrm{mmol})$, excess urea (55 equiv, $6.6 \mathrm{mmol}, 0.40 \mathrm{~g}$ ), and $12 \mathrm{~mL}$ dimethylformamide (DMF). The mixture was heated to reflux under nitrogen overnight. The reaction was judged incomplete via thin layer chromatography (TLC) in the morning and urea was added in 55 equivalent portions each hour (x 2) until all the starting material was consumed (if all 
starting material is not consumed, the reaction can still be worked up and the anhydride starting material recovered following purification). Once the reaction is judged complete, it is cooled, diluted with $\mathrm{CHCl}_{3}$ and washed with saturated aqueous $\mathrm{NH}_{4} \mathrm{Cl}(1 \times 30 \mathrm{~mL})$, brine $(1 \times 30 \mathrm{~mL})$ and the organic layer is dried over anhydrous sodium sulfate. Removal of solvent under reduced pressure followed by flash column chromatography $(100 \%$ $\mathrm{CHCl}_{3}$ ) yields $\mathbf{2 d}$ in quantitative yield as a dark purple solid. HR FAB found 912.5074 for $\mathrm{C}_{60} \mathrm{H}_{68} \mathrm{~N}_{2} \mathrm{O}_{6}$ (calc.912.5077). IR ( $\mathrm{CH}_{2} \mathrm{Cl}_{2}$ cast) 3163, 2958, 2850, 1697, 1604, 1259 $\mathrm{cm}^{-1}$. UV-vis (1.0 cm quartz cuvette, toluene) 401, 508, $545 \mathrm{~nm} .{ }^{1} \mathrm{H}$ NMR $\left(\mathrm{CDCl}_{3}, 500\right.$ MHz): $\delta 9.68(\mathrm{t}, 2 \mathrm{H}, J=8.85 \mathrm{~Hz}), 8.65(\mathrm{~d}, 1 \mathrm{H}, J=6.10 \mathrm{~Hz}), 8.63(\mathrm{~d}, 1 \mathrm{H}, J=6.10 \mathrm{~Hz})$, $8.45(\mathrm{~s}, 1 \mathrm{H}), 8.37(\mathrm{~s}, 1 \mathrm{H}), 8.36(\mathrm{~s}, 1 \mathrm{H}), 7.37(\mathrm{~s}, 1 \mathrm{H}), 7.36(\mathrm{~s}, 1 \mathrm{H}), 7.03(\mathrm{~d}, 2 \mathrm{H}, J=1.53$ $\mathrm{Hz}), 7.02(\mathrm{~d}, 2 \mathrm{H}, J=7.02 \mathrm{~Hz}), 4.15(\mathrm{t}, 2 \mathrm{H}, J=7.33 \mathrm{~Hz}), 1.34(\mathrm{~m}, 48 \mathrm{H}), 0.86(\mathrm{t}, 3 \mathrm{H}, J=$ $6.71 \mathrm{~Hz})$.

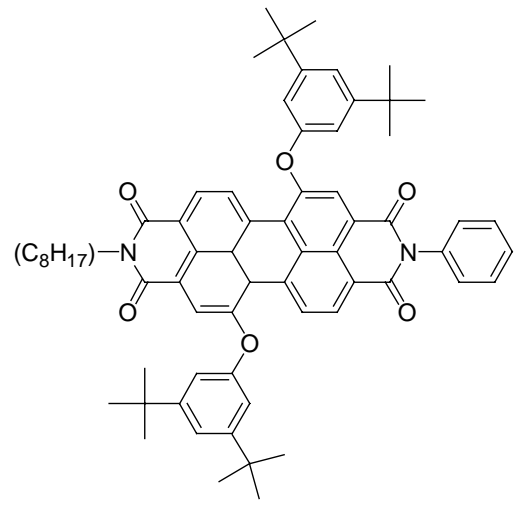

Compound 7, 5,12-Bis-(3,5-di-tert-butyl-phenoxy)-2-octyl-9-phenyl-5a,14c-dihydroanthra[2,1,9-def;6,5,10-d'e'f']diisoquinoline-1,3,8,10-tetraone.

As per general reaction conditions $(20 \mathrm{mg}, 0.022 \mathrm{mmol})$ of $\mathbf{2 d}$, (11 $\mathrm{mg}, 0.07$ $\mathrm{mmol}$ ) of arylboronic ester 1c, (12 mg, $0.07 \mathrm{mmol}$ ) of copper (II) acetate, and (7 mg, 0.07 mmol) of triethylamine were stirred at $45{ }^{\circ} \mathrm{C}$ for 48 hours in $2 \mathrm{~mL}$ chloroform. Purification via flash column chromatography $\left(100 \% \mathrm{CHCl}_{3}\right)$ yielded 7 as a dark purple solid (16 mg, 74\%). UV-vis (1.0 cm quartz cuvette, toluene) 403, 507, $543 \mathrm{~nm}$. HR FAB found 986.5230 for $\mathrm{C}_{66} \mathrm{H}_{70} \mathrm{~N}_{2} \mathrm{O}_{6}$ (calc. 986.5234). IR $\left(\mathrm{CH}_{2} \mathrm{Cl}_{2}\right.$ cast) 2922, 2852, 1697 , 1658, 1602, $1260 \mathrm{~cm}^{-1} .{ }^{1} \mathrm{H}$ NMR $\left(\mathrm{CDCl}_{3}, 500 \mathrm{MHz}\right): \delta 9.71(\mathrm{~d}, J=8.2 \mathrm{~Hz}, 2 \mathrm{H}), 8.68(\mathrm{~d}$, $J=8.2 \mathrm{~Hz}, 1 \mathrm{H}), 8.64(\mathrm{~d}, J=8.2 \mathrm{~Hz}, 1 \mathrm{H}), 8.34(\mathrm{~s}, 2 \mathrm{H}), 7.54(\mathrm{~m}, 2 \mathrm{H}), 7.47(\mathrm{~m}, 1 \mathrm{H}), 7.34$ $(\mathrm{m}, 4 \mathrm{H}), 7.04(\mathrm{~s}, 2 \mathrm{H}), 7.03, \mathrm{~s}, 2 \mathrm{H}), 4.15(\mathrm{t}, J=7.9 \mathrm{~Hz}, 2 \mathrm{H}), 1.30(\mathrm{~m}, 48 \mathrm{H}), 0.87(\mathrm{~m}, 3 \mathrm{H})$. 


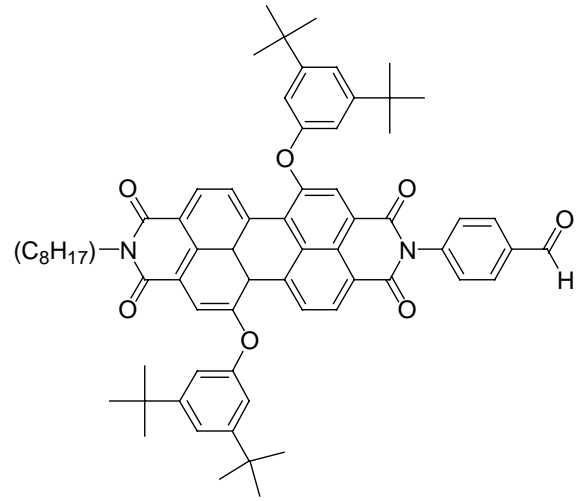

Compound 10, 4-[6,13-Bis-(3,5-di-tert-butyl-phenoxy)-9-octyl-1,3,8,10-tetraoxo3,5b,8,9,10,10c-hexahydro-1H-anthra[2,1,9-def;6,5,10-d'e'f']diisoquinolin-2-yl]benzaldehyde.

As per general reaction conditions $(20 \mathrm{mg}, 0.022 \mathrm{mmol})$ of $\mathbf{2 d}$, (13 $\mathrm{mg}, 0.07$ mmol) of 4-formylphenylboronate (9), (12 mg, $0.07 \mathrm{mmol})$ of copper (II) acetate, and (7 $\mathrm{mg}, 0.07 \mathrm{mmol}$ ) of triethylamine were stirred at $40{ }^{\circ} \mathrm{C}$ for 48 hours in $4 \mathrm{~mL}$ chloroform. Purification via flash column chromatography $\left(100 \% \mathrm{CHCl}_{3}\right)$ yielded $\mathbf{1 0}$ as a dark purple/red solid (15 mg, 73\%). HR FAB found 1016.5350 for $\mathrm{C}_{67} \mathrm{H}_{72} \mathrm{~N}_{2} \mathrm{O}_{7}$ (calc. 1016.5340). IR ( $\mathrm{CH}_{2} \mathrm{Cl}_{2}$ cast) $\left.2960,2854,1699,1659,1600,1262 \mathrm{~cm}^{-1}\right) . \mathrm{UV}$-vis $(1.0 \mathrm{~cm}$ quartz cuvette, toluene) 401, 509, $546 \mathrm{~nm} .{ }^{1} \mathrm{H} \mathrm{NMR}\left(\mathrm{CDCl}_{3}, 500 \mathrm{MHz}\right): \delta 10.11(\mathrm{~s}, 1 \mathrm{H})$, $9.69(\mathrm{~d}, 1 \mathrm{H}, J=8.55 \mathrm{~Hz}), 9.66$ (d, 1H, $J=8.55 \mathrm{~Hz}), 8.67$ (d, 1H, $J=8.24 \mathrm{~Hz}), 8.61$ (d, $1 \mathrm{H}, J=8.55 \mathrm{~Hz}), 8.22(\mathrm{~s}, 1 \mathrm{H}), 8.17(\mathrm{~s}, 1 \mathrm{H}), 8.06(\mathrm{~d}, 2 \mathrm{H}, J=7.94 \mathrm{~Hz}), 7.55(\mathrm{~d}, 2 \mathrm{H}, J=$ $8.24 \mathrm{~Hz}), 7.37$ (s, 1H), $7.36(\mathrm{~s}, 1 \mathrm{H}), 7.04$ (s, 2H), 7.02 (s, 2H), 4.13 (t, 2H, J = $7.63 \mathrm{~Hz})$, $1.33(\mathrm{~m}, 48 \mathrm{H}), 0.87$ (t, 3H, $J=6.10 \mathrm{~Hz})$. 


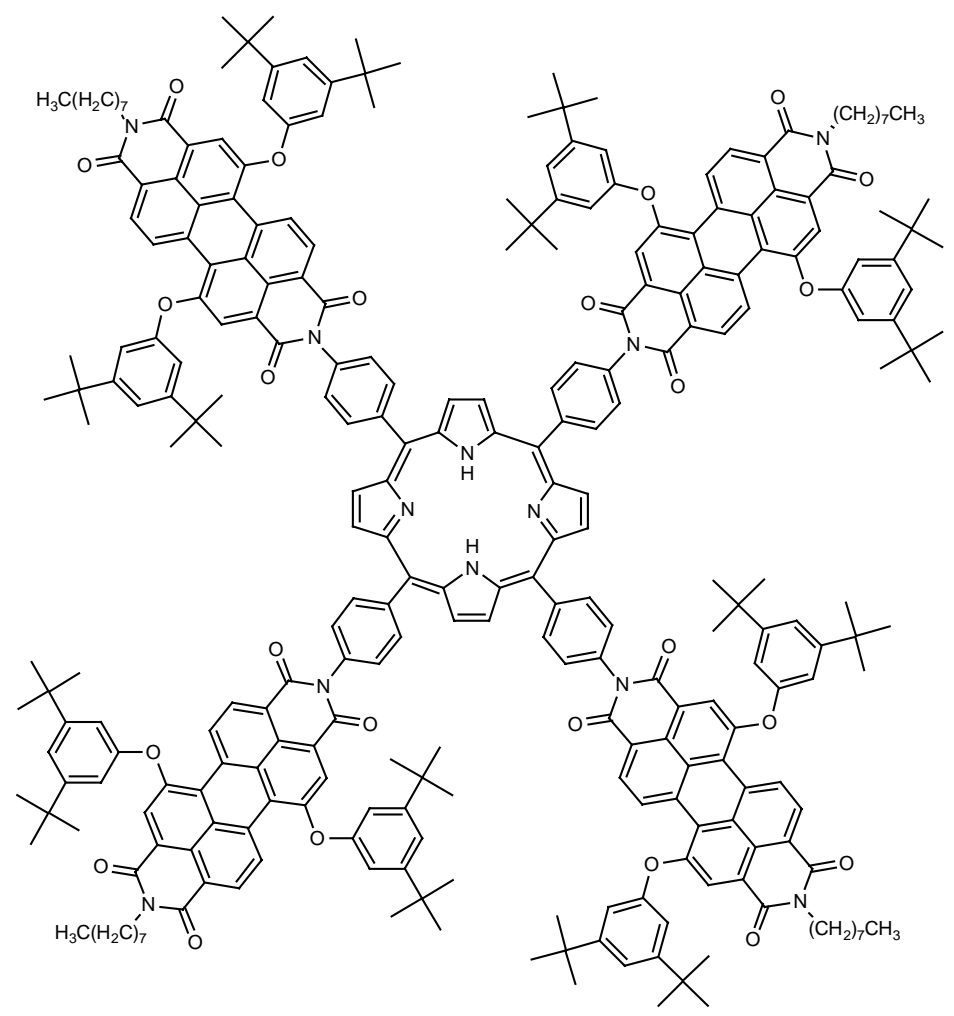

Compound 11, TPP-(PDI) ${ }_{4}$ [Tetraphenylphorphyrin-(perylenediimide) $\left.)_{4}\right]$

A $25 \mathrm{~mL}$ round bottom flask was flame dried and $10(21 \mathrm{mg}, 0.02 \mathrm{mmol})$, pyrrole (1.4 $\mathrm{mg}\left[0.47 \mathrm{~mL}\right.$ of a $3 \mathrm{mg} / \mathrm{mL}$ solution of pyrrole in $\left.\left.\mathrm{CH}_{2} \mathrm{Cl}_{2}\right], 0.02 \mathrm{mmol}\right)$ and $\mathrm{CH}_{2} \mathrm{Cl}_{2}(2 \mathrm{~mL})$ were added and degassed with nitrogen. Trifluoroacetic acid (TFA) $(4.7 \mathrm{mg}$ [0.62 mL of a $8 \mathrm{mg} / \mathrm{mL}$ solution of TFA in $\mathrm{CH}_{2} \mathrm{Cl}_{2}$ ], $0.04 \mathrm{mmol}$ ) was then added to the mixture and stirred at room temperature for 4 hours under a nitrogen atmosphere. $p$-Chloranil $(10 \mathrm{mg}$, $0.04 \mathrm{mmol}$ ) was added and the reaction heated to reflux for one hour under nitrogen. Purification via flash column chromatography ( $96 \% \mathrm{CHCl}_{3} / 4 \%$ EtOAc) followed by preparatory thin layer chromatography (gradient: $95 \% \mathrm{CHCl}_{3} / 5 \%$ Hexanes followed by 96\% $\mathrm{CHCl}_{3} / 4 \%$ EtOAc) yielded $\mathbf{1 1}$ as a dark purple solid (5 $\mathrm{mg}, 23 \%$ yield). UV-vis (1.0 cm quartz cuvette, toluene) $422,515,550 \mathrm{~nm} . \mathrm{C}_{284} \mathrm{H}_{294} \mathrm{~N}_{12} \mathrm{O}_{24}$. Mass spectrum (m/e) 4259.4 (calc.4259.4). IR ( $\mathrm{CH}_{2} \mathrm{Cl}_{2}$ cast) 3553, 2961, 2850, 1699, 1660, 1600, 1260, 1092, $1019,799 \mathrm{~cm}^{-1}$. Attempts to obtain ${ }^{1} \mathrm{H}$ NMR only yielded broad spectra due to the strong tendency of this molecule to aggregate as previously reported for a similar porphyrin (van der Boom, T.; Hayes, R. T.; Zhao, Y.; Bushard, P. J.; Weiss, E. A.; Wasielewski, M. R. J. Am. Chem. Soc. 2002, 124, 9582-9590.) ${ }^{1} \mathrm{H}$ NMR ( $\left.\mathrm{CDCl}_{3}, 500 \mathrm{MHz}\right): \delta 9.6$ (broad, $\left.8 \mathrm{H}\right)$, 
8.88 (broad, 4H), 8.8 (broad, 4H), 8.7 (broad, 4H), 8.4 (broad, 4H), 7.7 (broad, 8H), 7.5 (broad 24H), 7.2 (broad, 24H), 4.2 (broad, 8H), 1.9 (broad, 16H), 1.4 (broad, 188H), -2.8 (broad, 2H).

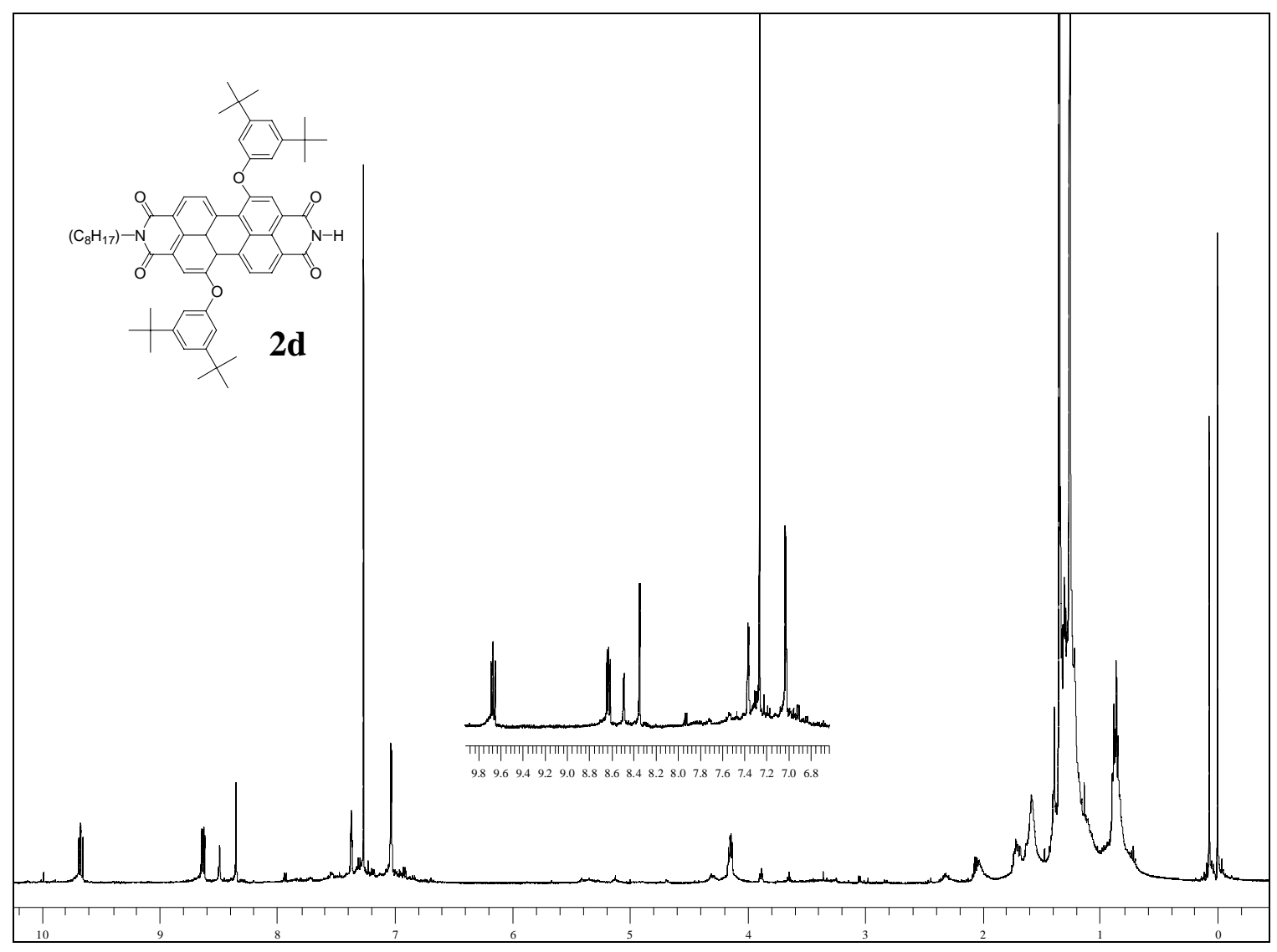




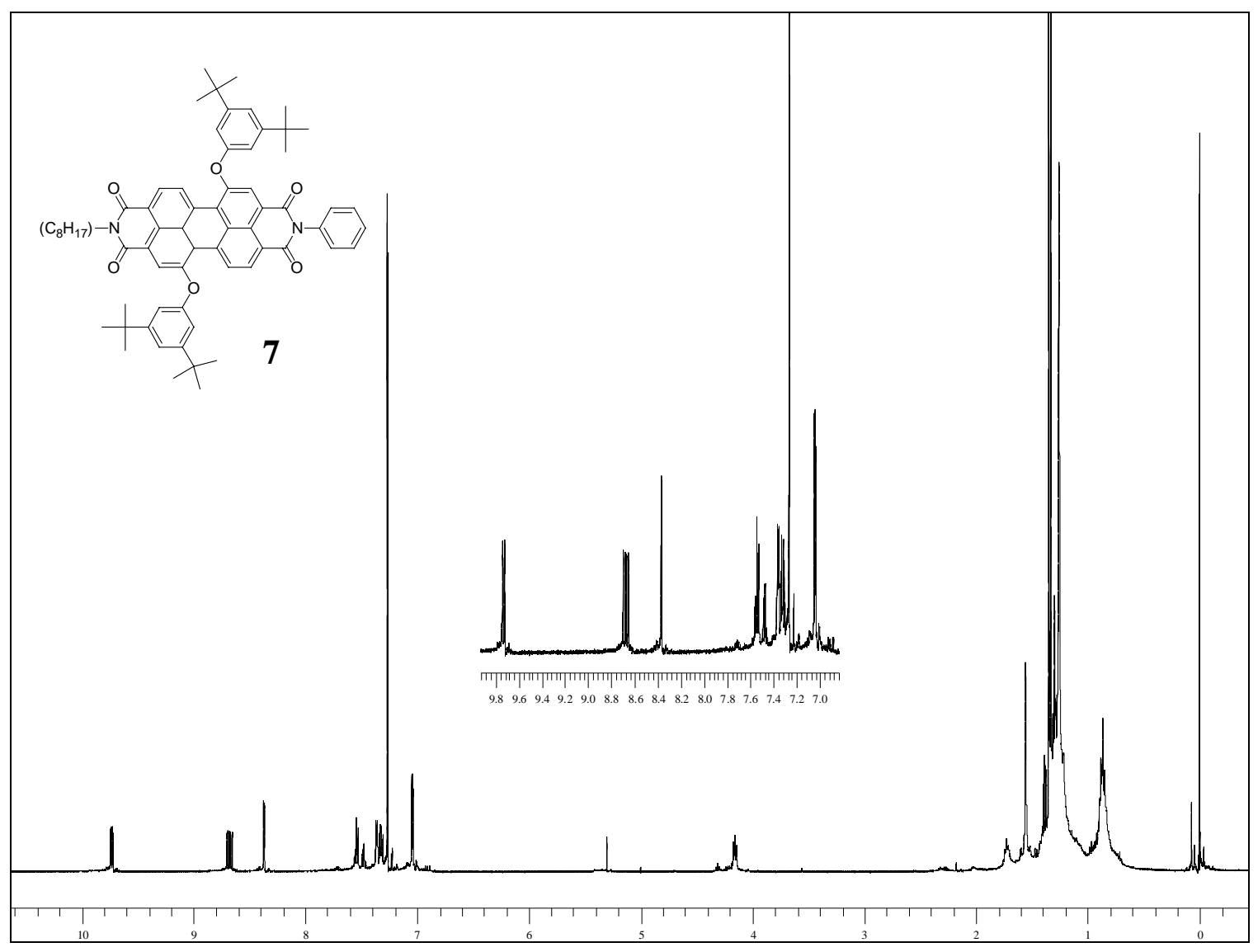




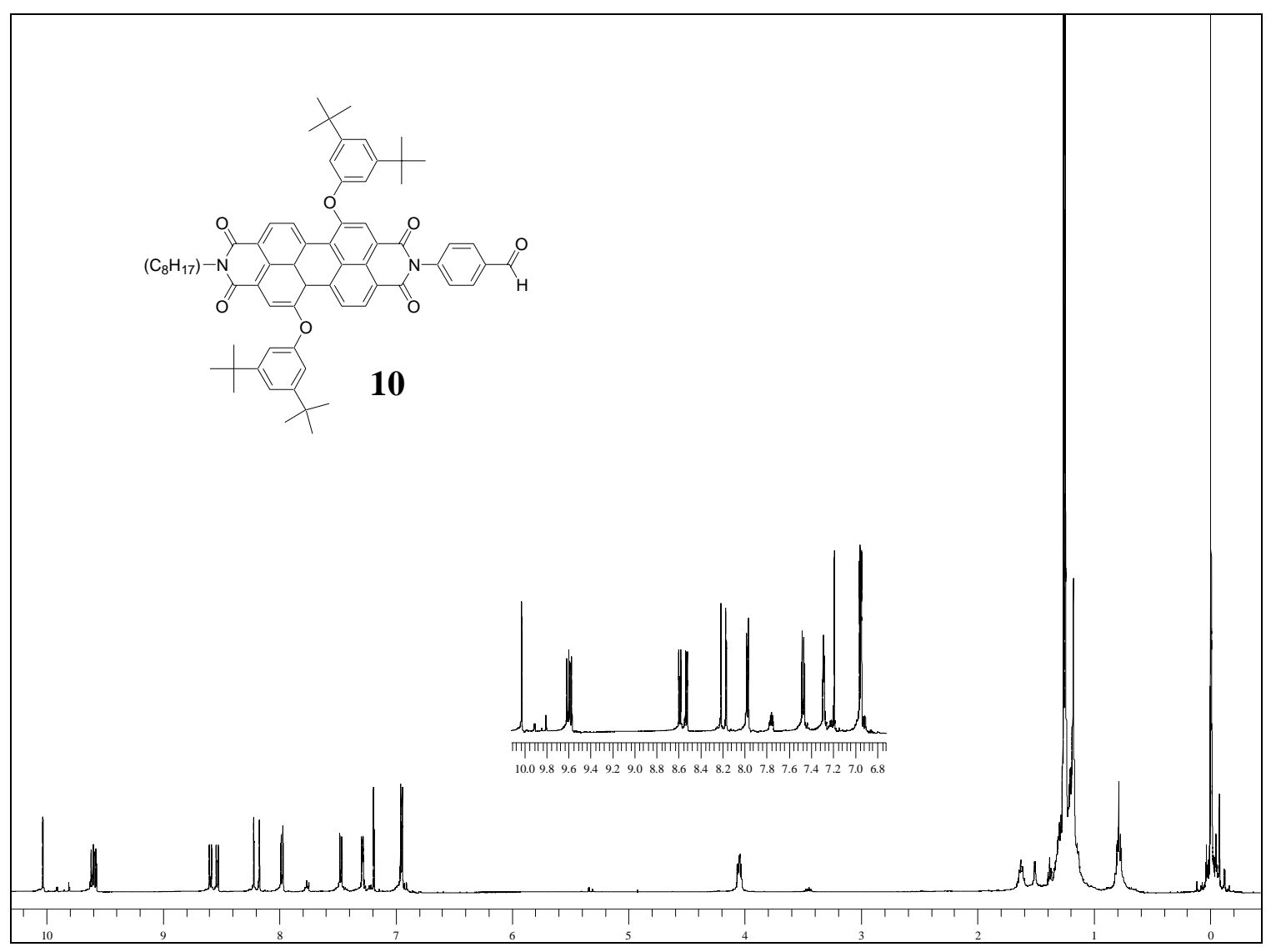




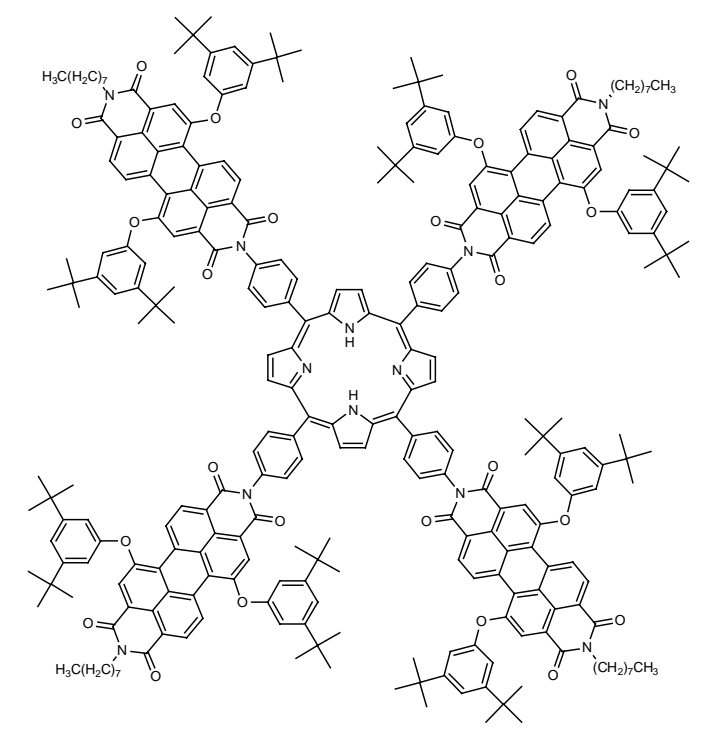

11

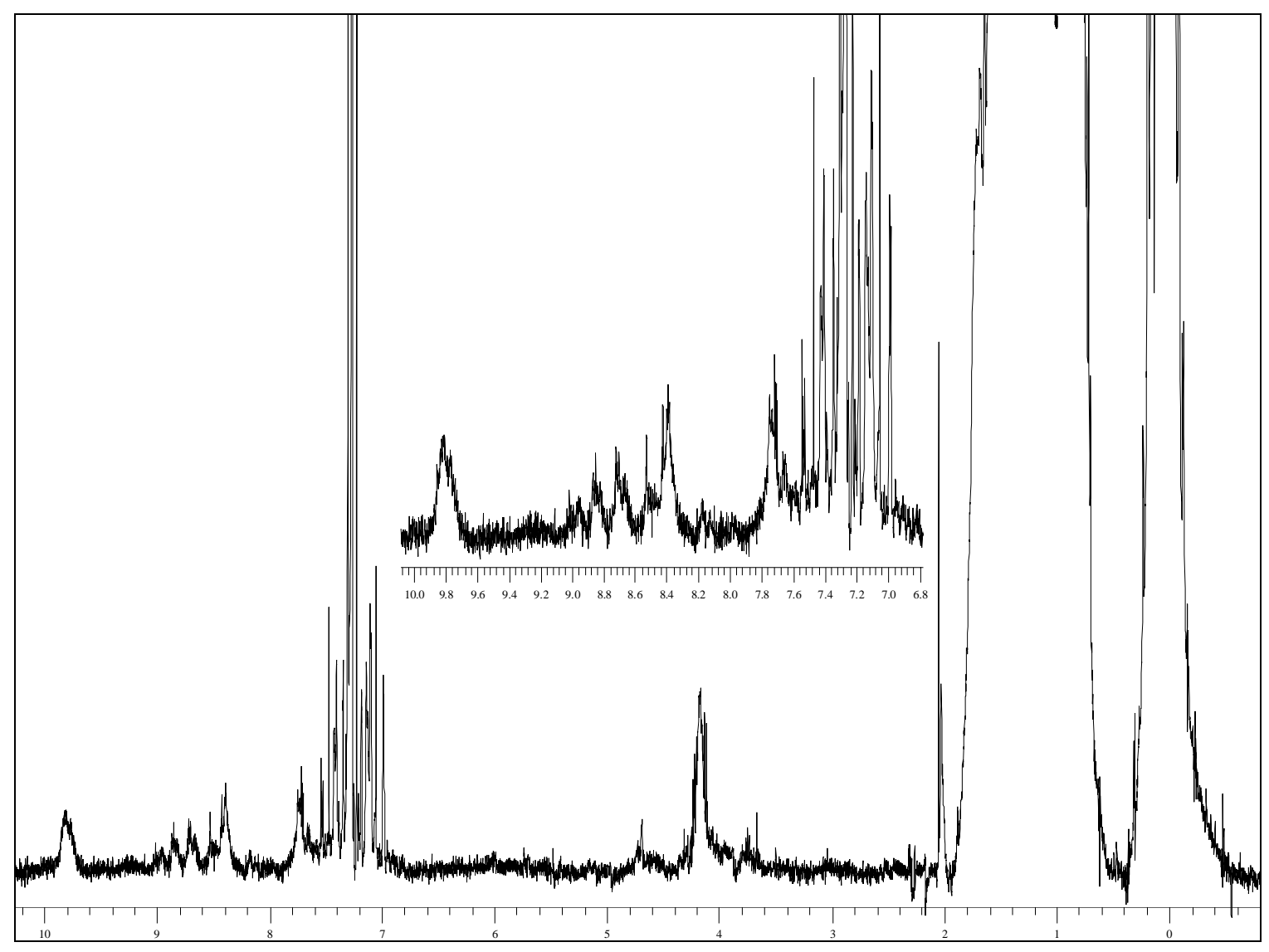




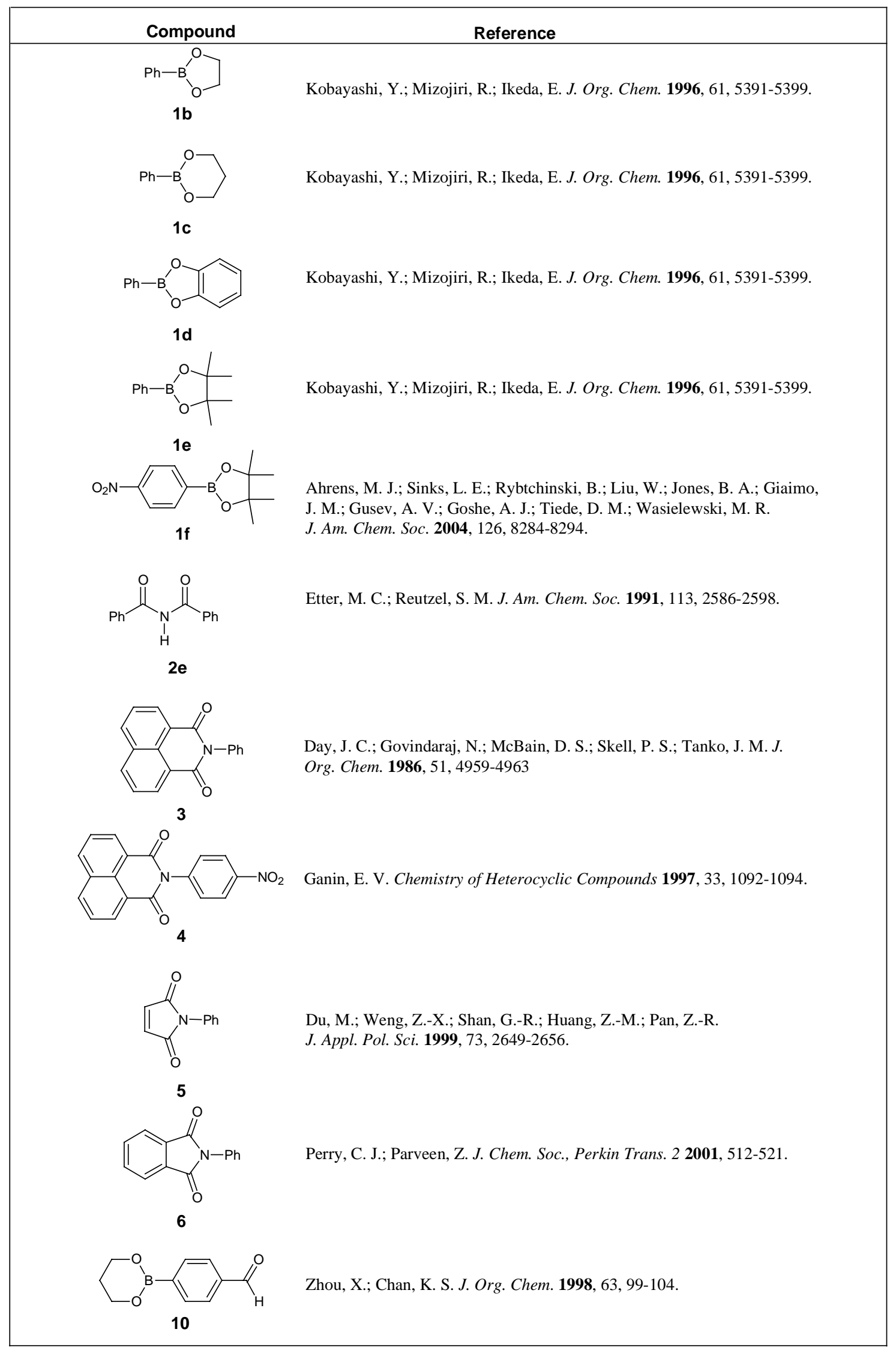

